\title{
Performance of a Large Scale Prototype of the ATLAS Accordion Electromagnetic Calorimeter
}

\section{The RD3 Collaboration}

D.M.Gingrich, G.Greeniaus, P.Kitching, B.Olsen, J.L.Pinfold and N.L.Rodning Alberta University, Edmonton, Alberta, Canada

E.Boos and B.O.Zhautykov

HEPI, Alma-Ata, Kazakhstan

B.Aubert, A.Bazan, B.Beaugiraud, J.Boniface, J.Colas, S.Jezequel, T.Leflour, M.Maire, F.Rival, M.Stipčevićc ${ }^{1)}$, J.Thion, D.VanDenPlas,

I.Wingerter-Seez, R.Zitoun and Y.P.Zolnierowski

$$
\text { LAPP, Annecy, France }
$$

M.Chmeissani, E.Fernandez, L1.Garrido, M.Martinez and C.Padilla

Univ. Autònoma Barcelona, Bellaterra, Spain

H.A.Gordon, V.Radeka, D.Rahm, and D.Stephani

Brookhaven National Laboratory, Upton, USA

L.Baisin, J.C.Berset, J.L.Chevalley, F.Gianotti ${ }^{2)}$, O.Gildemeister, C.P.Marin, M.Nessi, L.Poggioli, W.Richter and V.Vuillemin CERN, Geneva, Switzerland

J.M.Baze, L.Gosset, P.Lavocat, J.P.Lottin, B.Mansoulié, J.P.Meyer, J.F.Renardy, J.Schwindling and J.Teiger

CEA, DSM/DAPNIA/SPP, CE Saclay, Gif-sur-Yvette, France

J.Collot, P.de Saintignon, D.Dzahini, J.Y.Hostachy, G.Laborie, G.Mahout, E.Merchez and J.Pouxe ISN, Grenoble, France

L.Hervas

Univ. Autònoma Madrid, Spain

A.Chekhtman, M.C.Cousinou, P.Dargent, B.Dinkespiller, F.Etienne,

P.Fassnacht, D.Fouchez, L.Martin, A.Miotto, E.Monnier, E.Nagy,

C.Olivetto and S.Tisserant

CPP Marseille, France

G.Battistoni, D.V.Camin, D.Cavalli, G.Costa, L.Cozzi, A.Cravero, N. Fedyakin, A.Ferrari, L.Mandelli, M.Mazzanti, L.Perini and P.Sala

Dipartimento di Fisica dell'Università e Sezione INFN, Milano, Italy

\footnotetext{
1) On leave from Rudjer Bošković Institute, Zagreb, Croatia

2) Also University of Milano, Italy
} 
G.Azuelos, G.Beaudoin, P.Depommier, E.León-Florián, C.Leroy and P.Roy University of Montreal, Canada

\author{
M.Seman \\ Nevis Laboratories, Columbia University, Irvington NY, USA \\ E.Augé, R.Chase, J.C.Chollet, C.de La Taille, L.Fayard, D.Fournier, \\ A.Hrisoho, B.Merkel, J.M.Noppe, G.Parrour, P.Pétroff, A.Schaffer, \\ N.Seguin-Moreau, L.Serin, V.Tisserand and I.Vichou \\ $L A L$, Orsay, France \\ B.Canton, J.David, J.F.Genat, D.Imbault, O. Le Dortz, \\ A.Savoy-Navarro and P.Schwemling \\ LPNHE, Universites de Paris VI et VII, France \\ L.O.Eek, B.Lund-Jensen and J.Söderqvist \\ Royal Institute of Technology, Stockholm, Sweden \\ M.Lefebvre ${ }^{3)}$, S.Robertson and J.White \\ University of Victoria, British Columbia, Canada
}

3) Also visiting scientist at LAL, Orsay, France 


\section{Abstract}

A 2 metre long prototype of a lead-liquid argon electromagnetic calorimeter with accordionshaped electrodes, conceived as a sector of the barrel calorimeter of the future ATLAS experiment at the LHC, has been tested with electron and pion beams in the energy range 10 to $287 \mathrm{GeV}$. A sampling term of $10 \% / \sqrt{E(\mathrm{GeV})}$ was obtained for electrons in the rapidity range $0<\eta<1$, while the constant term measured over an area of about $1 \mathrm{~m}^{2}$ is $0.69 \%$. With a cell size of $2.7 \mathrm{~cm}$ the position resolution is about $4 \mathrm{~mm} / \sqrt{E(\mathrm{GeV})}$. 
The electromagnetic calorimeter of the future ATLAS experiment at the LHC [1, 2] will combine the liquid argon (LAr) sampling technique with an accordion geometry and a fast readout. This technology has been pioneered since 1990 by the RD3 Collaboration, which demonstrated the feasibility of the Accordion concept by successfully testing, with beams of particles, small size prototypes with non-projective and semi-projective geometry $[3,4,5]$.

As a further step towards the future detector, the RD3 Collaboration has built a larger scale prototype with a fully pointing structure, already conceived as a sector of the ATLAS calorimeter. This prototype has been tested with high energy electron and pion beams at the CERN Super Proton Synchrotron (SPS) together with a LAr hadronic calorimeter prototype which also had an Accordion geometry [6]. The main points to be investigated in the electromagnetic part were the capability of maintaining the energy resolution at the level of $10 \% / \sqrt{E(\mathrm{GeV})}$ over a large angular coverage and the impact of the mechanics, of the readout and of the calibration system of a large detector on its performance and uniformity of response.

These and other questions are the object of this paper, which is organized as follows. Section 2 describes the mechanical structure, the readout and the calibration system of the calorimeter prototype. The experimental setup for the beam tests is illustrated in section 3 , while sections 4 to 6 present the main results from the data analysis with particular emphasis on the energy resolution and on the uniformity of response. Finally section 7 is devoted to the conclusions.

\section{The Calorimeter Prototype}

The large scale Accordion prototype is a cylindrical sector consisting of three mechanically independent azimuthal modules. It extends over $2 \mathrm{~m}$ along the $z$ axis ${ }^{1)}$, which corresponds to the pseudorapidity range $0 \leq \eta \leq 1.08$, and covers $27^{\circ}$ in $\phi$. The total acceptance represents about $3 \%$ of the future barrel calorimeter of ATLAS. The prototype is fully pointing towards the nominal LHC interaction centre and has an inner radius of $131.5 \mathrm{~cm}$ and an outer radius of $182.6 \mathrm{~cm}$.

Figure 1 shows the detector during assembly. The stack consists of 73 converter plates interleaved with 72 readout electrodes. Both have an accordion shape with 18 folds and 17 straight sections. The total thickness is $25 X_{0}$ at $\eta=0$, divided into three longitudinal compartments (front, middle and back) of $9 X_{0}, 9 X_{0}$ and $7 X_{0}$ respectively.

The $2 \mathrm{~m}$ long absorbers are made of $\mathrm{Pb}$ plates $(1.8 \mathrm{~mm}$ thick for $\eta<0.7$ and $1.2 \mathrm{~mm}$ thick in the rest of the coverage), which are sandwiched in between two $0.2 \mathrm{~mm}$ stainless steel sheets to ensure a higher mechanical strength. The electrodes are multilayer copper-kapton boards of thickness $0.3 \mathrm{~mm}$, separated from the converter plates by a $1.9 \mathrm{~mm}$ LAr gap. The basic sampling cell of the calorimeter is shown in fig. 2 .

Three consecutive kapton boards are grouped together in the same channel at the readout level, giving an effective granularity $\Delta \phi \simeq 0.020$, while the transverse segmentation $\Delta \eta \simeq$ 0.018 ( $\Delta \eta \simeq 0.036$ in the back compartment) was obtained by etching projective strips on the conductive layers of the electrodes.

The main constraint on the design of the detector was dictated by the full projectivity of the structure, which had to be combined with the requirement of having a LAr gap (and therefore a sampling fraction) constant with the calorimeter depth. The $\phi$-symmetry was simply achieved by repeating the absorber and electrode plate structure around the collider beam line, with an angle of $2 \pi / 960$ between adjacent absorber plates, and with the folds running parallel to the beam axis. The thickness of the LAr gap and the sampling fraction were kept constant by gradually changing the angle of the accordion folds along the depth of the calorimeter from $87^{\circ}$ to $111^{\circ}$ (fig. 1). A further constraint on the geometrical parameters of the detector was the uniformity of the amount of LAr traversed as a function of $\phi$, which is directly related to

\footnotetext{
1) In the collider reference system adopted here the $z$ axis indicates the LHC beam line while $\phi$ and $\theta$ are the
} azimuthal and polar angle respectively. The pseudorapidity is defined as $\eta=-\ln \tan (\theta / 2)$. 
the intrinsic response uniformity of the calorimeter. The final mechanical layout was therefore guided by a detailed Monte Carlo simulation of the Accordion [7].

As demonstrated by the tests of previous prototypes, the adopted sampling structure allows to achieve an energy resolution of typically $10 \% / \sqrt{E(\mathrm{GeV})}$. However at large rapidity the contribution to the energy resolution from the sampling fluctuations is expected to increase as $1 / \sqrt{\sin \theta}$, due to the decrease of the sampling frequency. Since the sampling term of the energy resolution scales also as $\sim \sqrt{t_{\mathrm{abs}}}$, where $t_{\mathrm{abs}}$ is the thickness of the converter plates expressed in radiation lengths, the calorimeter performance can in principle be kept at the same level over its full acceptance by suitably reducing the density of the absorber. Therefore in the 2 metre prototype the lead thickness in the converter plates was reduced from $1.8 \mathrm{~mm}$ to $1.2 \mathrm{~mm}$ for $\eta \geq 0.7$.

\subsection{Readout electrodes}

The readout electrodes are multilayer boards consisting of two (one double-sided and one single-sided) copper clad kapton sheets glued together. The copper and kapton foils are $35 \mu \mathrm{m}$ and $25 \mu \mathrm{m}$ thick respectively. The three copper layers are etched into projective strips, separated by a $1 \mathrm{~mm}$ gap for insulation, which define the longitudinal and the $\eta$ segmentation of the detector.

The ionization charge drifting in the LAr gap induces by capacitive coupling a signal on the inner conductive layer which is DC coupled to the input of the preamplifier, the first element of the readout chain. The two outer conductive layers are at high voltage $(2 \mathrm{kV})$ and produce an electric field of about $10 \mathrm{kV} / \mathrm{cm}$ over the gap. In order to protect the preamplifiers from accidental sparking, these layers consist of alternating pads of copper and resistive coating. This solution was preferred to a uniform coated facesheet because it ensures a higher rigidity of the board in the bent regions.

The front and back parts of the kapton circuits consist of a flat fibreglass section ( 0.8 $\mathrm{mm}$ thick) containing routing for the high voltage distribution and for the output signals. It also houses a set of holes needed to mount and fix the electrodes to the support bars in the calorimeter stack (see section 2.3).

While a converter plate extends over the full detector length, for ease of fabrication five different electrode boards, each one of size $\Delta \eta=0.216$, were used to cover the calorimeter acceptance in $\eta$.

Due to a fault in the design of the electrode layout, a capacitive coupling between channels of the middle and back compartment gave rise to a few percent level crosstalk, which was corrected offline channel by channel by using calibration data. A batch of new modified electrodes will equip part of the detector in the next beam tests.

\subsection{Absorber plates}

The fabrication procedure of accordion shaped converter plates has already been described elsewhere [3]. Lead and stainless steel sheets were first cut to the desired shape and cleaned by chemical and mechanical processes. They were then stacked together as a sandwich which, in a following phase, was bent to the accordion shape. At $\eta<0.7$ the sandwiches consist of five elements (stainless steel, "prepreg adhesive" 2), lead, prepreg adhesive, stainless steel), while at large rapidity (thinner $\mathrm{Pb}$ thickness) two more stainless steel foils and prepreg layers were inserted to leave the overall plate thickness unchanged. To ensure the highest uniformity of the absorbers, dedicated precision tooling were built and maximum care was taken during the plate fabrication. Plates were sorted in such a way that the overall r.m.s. dispersion of the lead thickness from plate to plate within a module measured before bending was about $0.01 \mathrm{~mm}$.

To bend the converters to the desired accordion shape, two independent set of dies, positioned on a fixed frame, were first mildly pressed on the plates in correspondence with the

2) Brochier $1454 \mathrm{NC} / 50 \% / 759$ 
bending corners. They were then released and the final pressing took place. In this phase dies moved on rollers closer and closer to each other as the vertical pressure (up to a maximum value of 5 bars) was applied.

The absorbers were then heated under pressure in a precisely shaped mould to polymerize the preimpregnated tissue layers until the final gluing was reached. First the temperature was ramped up at a rate of $3{ }^{\circ} \mathrm{C}$ per minute, then it was maintained for an hour at $120{ }^{\circ} \mathrm{C}$ and with a pressure of 5 to 6 bars. Finally the temperature was ramped down at the same rate. After the heating and pressing cycle the thickness of the prepreg layers was on average $0.13 \mathrm{~mm}$. These layers are dry preimpregnated clothes with glue on one side only which during heating flows through the fibre frame. It is important that in the initial phase of the bending procedure the connecting surfaces, lead and stainless steel, can easily slide on each other.

At the end of the gluing process the mean thickness of the converter plates was 2.49 $\mathrm{mm}$ with an r.m.s. deviation of $0.05 \mathrm{~mm}$. The plate deviations from flatness were within 0.1 $\mathrm{mm}$ longitudinally and between 0.3 and $0.5 \mathrm{~mm}$ transversally. This residual sagging of "free standing" plates is to a large extent removed by fixture to the precision bars (see below). In order to minimize the plate to plate differences in each module, the converters were mounted in the stack according to the thickness of the lead, as shown in fig. 3 .

Before stacking each converter was equipped, on both sides, with $3 \mathrm{~cm}$ wide honeycomb bands running along the $2 \mathrm{~m}$ dimension and glued to the plate in a few points. Their function is to maintain the readout electrode centered within the LAr gap.

\subsection{Detector assembly}

The cylindrical symmetry of each module and the correct gap thickness between the plates were obtained by gluing ${ }^{3}$ each absorber, both at the front and at the back side, to wedge shaped fibreglass (G10) precision bars, which were then piled on each other. The bars are $2 \mathrm{~m}$ long and extend over $20 \mathrm{~mm}$ in the radial direction. Their thickness was controlled to better than $10 \mu \mathrm{m}$ to ensure a correct gap within $\pm 50 \mu \mathrm{m}$. The glass fibres in the bars were aligned azimuthally and along the $2 \mathrm{~m}$ dimension, to ensure a similar thermal contraction of the various detector components in the transverse direction. Notches in the outer side of the bars allow for the kapton electrodes feedthrough and positioning. The grooves in the bars are wide enough to allow for a correct positioning of the elements without building internal constraints at those places.

Temporary rods interleaved in between the converter and readout electrodes were used during stacking to maintain the gap thickness and to prevent sagging effects under gravity. At the end of the stacking each module was compressed by using tie-rods penetrating all the G10 bars and ensuring the necessary rigidity to the pile. The temporary rods were then removed.

After assembly the mechanical uniformity of the stack was estimated by measuring the capacitances $\left(\mathrm{C}_{\mathrm{gap}}\right)$ of several double gaps, each one consisting of one electrode and the two absorber plates on its opposite sides. If $d$ is the distance between two consecutive converters and $C_{1,2}$ is the capacity of the two single gaps, then

$$
C_{\text {gap }}=C_{1} \| C_{2}=C_{1}+C_{2} \propto \frac{1}{d / 2-\epsilon}+\frac{1}{d / 2+\epsilon} \simeq \frac{4}{d}
$$

where $\epsilon$, the displacement of the electrode from the centre of the double gap, is supposed to be negligible with respect to $d$. The measurements were performed at room temperature in a frequency region $(>100 \mathrm{kHz})$ where the resistive impedance effects become negligible. All $\phi$ cells of four different $\eta$ rows were tested, for a total of 288 measurements. The results are shown in fig. 4 . At the transition between two azimuthal modules the measured capacity is $6-7 \%$ lower than the average, thus indicating a larger LAr gap. Excluding these discontinuities, the resulting r.m.s. of the capacitances is $1.4 \%$, which means a dispersion of about $55 \mu \mathrm{m}$ in the thickness of the double gaps.

\footnotetext{
3) Araldite AW 106 was used.
} 
After two years since the assembly of the detector and after four cool down cycles for beam tests, one module was dismantled and inspected. No sign of ungluing of the absorber sandwiches or of the G10 bars was observed.

\subsection{Readout electronics}

The three different options adopted for the front-end electronics were already tested with previous prototypes and described in $[4,5]$ : hybrid charge preamplifiers working at LAr temperature and based on Silicon JFETs [8] or GaAs MESFETs [9] and warm current preamplifiers (the so called "0T" scheme, meaning no transistor in the cold [10]) placed outside the cryostat.

To allow for a fast charge transfer in the first two cases, the preamplifiers were placed directly on printed circuit motherboards, which were mounted on the front and back face of the calorimeter and contained also the calibration system. The preamplifier input impedance was typically $10 \Omega$ and the power dissipated in the cold $54 \mathrm{~mW}(72 \mathrm{~mW}$ ) for the GaAs (Si) hybrids.

In the $0 \mathrm{~T}$ scheme the signal is sent from the calorimeter to a warm current preamplifier placed outside the cryostat through a $50 \Omega$ cable. The main advantages of this solution are an easier access for maintenance, a better shielding from radiation and a negligible power dissipation in the cold. By a careful shielding of the cable [10] the larger sensitivity of this scheme to pickup noise can be kept under control.

The preamplifier output was shaped outside the cryostat with a $C R^{2} R C^{3}$ bipolar filter in case of a charge preamplifier and with a $C R R C^{2}$ unipolar filter in case of a current preamplifier. The peaking time (between $5 \%$ and the peak) of the shaped response to a $\delta$-current pulse was $t_{p}(\delta)=18 \mathrm{~ns}$, corresponding to about $35 \mathrm{~ns}$ for the triangular ionization signal from the detector. This shaping time was chosen so as to minimize the total noise expected in the calorimeter from the electronics and from the pile-up of several soft interactions in the preceeding and following bunch crossings under LHC operating conditions.

The shapers were followed by track and hold $(\mathrm{T} \& \mathrm{H})$ circuits, whose hold signal was generated by a beam concidence or by a calibration trigger and whose timing was adjusted to sample the shaper signal at its maximum. The output was digitized by a 12-bit charge integrating ADC.

In each longitudinal sampling of the calorimeter the readout was organized in 15 blocks: 3 modules in $\phi$ and 5 sectors in $\eta$, the latter corresponding to the 5 different kapton electrodes. Each block contained $12 \times 8$ cells in $\eta \times \phi$ in the first two samplings and $6 \times 8$ in the last one. For the beam test only 2000 out of 3600 channels were fully equipped.

\subsection{Calibration}

In order to exploit the good intrinsic energy resolution of the detector, an excellent calibration system is needed. It must be able to correct for the different electronic gains of the various channels, to measure possible crosstalk levels and to monitor the stability of the readout chain during long periods of data taking.

Two new calibration systems have been tested with the 2 metre prototype: a "voltage driven" and a "current driven" system. The lack of space for the hybrids of the current driven calibration and their preliminary status of development led to equip the whole detector with the voltage calibration, for which there is only a resistor in the liquid. However in the 0T region, where there are no preamplifiers in the cold and there is therefore more space, both calibration systems were installed in order to compare their respective accuracy and merits.

The calibration hardware and procedure are discussed in detail in the next paragraphs.

\subsubsection{Voltage driven calibration}

In this case the only element in the liquid is a $2 \mathrm{k} \Omega$ injection resistor, the voltage pulse simulating the detector signal being brought from outside the cryostat. There are some advantages in calibrating with resistors: they are easily within $0.1 \%$ precision, they are reliable

and radiation hard. With fast shaping $\left(t_{p}(\delta) \simeq 20 \mathrm{~ns}\right)$ and relatively high detector capacitance $\left(C_{d} \simeq 400 \mathrm{pF}\right)$ the parallel noise they generate is small compared to the series noise from the preamplifier $\left(\mathrm{ENC}_{p}=700 e^{-}\right.$compared to $\left.\mathrm{ENC}_{s}=15000 e^{-}[11]\right)$. 
The main problem resides in the distribution of the precision voltage pulse, which has to be uniform (the design goal is within $0.2 \%$ ) throughout the calorimeter. To reduce the number of cables, each calibration signal is distributed locally to channels which have no or negligible physical crosstalk. However these channels should not be too far apart from each other as the calibration should remain synchronous with respect to the particle arrival. It is also important that all the elements in the calibration path be as uniform as possible (within $0.1 \%$ ). Among those elements, the cable that brings the signal down to the towers is of major concern. On one hand, it can be shown that when the cable is terminated at both ends, the output voltage is a second order function of the cable impedance [12]. For example a $10 \%$ error on the cable characteristic impedance would result in a $0.25 \%$ error on the pulse amplitude (provided that the cable is longer than the shaping time, so that reflected signals do not contribute to the shaped amplitude). On the other hand signal attenuation due to skin effects in the cable is a first order effect, so that all conductor dimensions in the cable should be as uniform as possible. It has been shown by measurements on the $0 \mathrm{~T}$ electronics that the attenuation is substantially reduced when the cable is cold [13].

The voltage pulse is generated by specially designed pulsers, that can drive up to $5 \mathrm{~V}$ into a $50 \Omega$ load with a risetime less than $1 \mathrm{~ns}$ and an exponential decay of $400 \mathrm{~ns}$ simulating the shape of the signal from the detector. It is obtained by switching off a precise DC current $I_{p}$ flowing in an inductor (fig. 5). A clock pulse is applied to the base of Q2 which diverts $I_{p}$ to ground, cutting Q1 off. The magnetic energy stored in the inductor is transferred to the resistance $R_{\mathrm{o}}$ and to the output cable, producing a voltage:

$$
V(t)=-\frac{R_{\mathrm{o}}}{2} I_{p} \exp (-t / \tau) \quad \text { with } \tau=\frac{L}{2 R_{\mathrm{o}}}
$$

The fact that the inductor exhibits a low resistance leads to low power dissipation in the pulser and in the terminating resistor $R_{0}$, as the collector of Q1 operates close to $0 \mathrm{~V}$, thus ensuring long term stability. However0 it does not affect the accuracy of the pulse initial amplitude, which is determined only by $I_{p}$ and $R_{\mathrm{o}}$. To ensure good linearity the base current of Q1, which varies non linearly with $I_{p}$ (as the transistor gain $\beta$ varies with collector current and temperature), is sensed and added to $I_{p}$. For the tests 12 channels have been implemented on a CAMAC board, to match an existing 12 conductor cable. This is a shielded $50 \Omega$ flat cable ${ }^{4}$ designed for the output signals [13]. The DAC and the clock inputs are common to the 12 channels, which can be separately enabled via the CAMAC bus. All the channels are synchronized within 0.2 ns and uniform within $0.2 \%$.

To reduce the number of pulsers each channel is split into four $100 \Omega$ cables, to drive four motherboards. Each $100 \Omega$ cable feeds an injection network (hybrids produced by Sfernice) which supports six $2 \mathrm{k} \Omega$ injection resistors $\left(\mathrm{R}_{t}\right)$ as well as decoupling capacitors to isolate the DC bias of the preamplifiers and a $143 \Omega$ resistor to ground to terminate the $100 \Omega$ cable in its characteristic impedance. The injection network drives one preamplifier out of every four in $\eta$, to allow for crosstalk studies, and two contiguous lines in $\phi$. Thus 24 preamplifiers are pulsed simultaneously from one pulser channel: 3 along $\eta$ and 8 along $\phi$. The other preamplifiers in the $\eta$ direction are pulsed with three other channels. The paths on the motherboards from the injection networks to the preamplifier inputs have been adjusted so that the calibration signals remain synchronous with particles arriving from a common LHC interaction vertex.

\subsubsection{Current driven calibration}

In this case only a DC current is sent from outside the cryostat and the calibration (current) pulse is generated near the preamplifier input with a long tailed pair (fig. 6 and [14]).

Si JFETs have been used, since p-channel devices are needed. In addition they operate at LAr temperature and have shown so far good radiation hardness when cold. Initially Q1 is on, Q2 is off and the programmed current $I_{p}$ flows into Q1 and $R_{t}$. The voltage across $R_{t}$ charges

\footnotetext{
4) manufactured by AXON, Montmirail, France.
} 
$C_{t}$ with $Q_{\circ}=R_{t} C_{t} I_{p}$. The clock signal turns on Q2 switching the current to ground and turning Q1 off. $C_{t}$ is then discharged through $R_{t}$, producing an exponential decay:

$$
Q=Q_{\circ} \exp \left(-t / R_{t} C_{t}\right)
$$

The current flowing into a preamplfier is thus:

$$
I_{t}=-\frac{d Q}{d t}=-I_{p} \exp \left(-t / R_{t} C_{t}\right)
$$

It stems from this that the initial current depends only on $I_{p}$ which can be made very precise as it is a DC current. The resistor and capacitor determine only the decay time, which should match the drift time, but within a few percent precision only, as it is a second order contribution to the signal amplitude at fast shaping.

To minimize clock injection it is preferable to switch off the calibration current, which necessitates the use of $p$-channel transistors in order to generate a negative calibration pulse. The clock signal must also be very fast not to degrade the signal risetime. A very attractive feature of this system is that it requires no precise components in the calibration path, except for a DC current $I_{p}$. There is no hard constraint on the clock signal which is only a digital signal, except its fast risetime, and it can also be distributed to many channels, as described further. Care must be taken to minimize capacitive coupling between this fast rising clock and the detector, as this results in a pedestal offset at zero calibration current. However this injected charge appears as the derivative of the real signal ( $\delta$ current compared to nearly a step) so that its contribution more or less passes through zero at the signal maximum.

In practice, two channels are mounted on one hybrid, thus sharing $I_{p}$, clock and bias. To be able to use the same 12 conductor AXON cable, the channels were multiplexed using six select lines, four current sources and two clocks per motherboard. The two clocks drove the two lines in $\phi$ independently and the preamplifiers along $\eta$ were activated individually through the select lines. The clock propagation time was designed to be $1 \mathrm{~ns}$ between two hybrids. It had to be compensated for externally with a precision delay generator according to the position of the pulsed preamplifier.

\subsubsection{Comparison between the two systems}

Uniformity measurements have been performed in a test bench in the laboratory on five motherboards from the detector region equipped with 0T preamplifiers, where both calibration systems were installed in parallel. A single channel readout chain was moved around the 24 channels of a motherboard. The readout chain was made up of a $0 \mathrm{~T}$ preamplifier, a shaper, a $\mathrm{T} \& \mathrm{H}$ and a 12-bit ADC, similar to those used in the beam test. The detector was simulated by 24 capacitors equal to within $0.2 \%$.

As only one motherboard was measured at a time on the test bench, the splitter and the $100 \Omega$ cables have not been used for the voltage calibration and the signal from the pulser was brought directly into the injection networks. The results (fig. 7 ) show an r.m.s. value of $0.33 \%$. Each motherboard has an r.m.s. around $0.3 \%$ and the dispersion between motherboards is small, indicating that the non uniformity is dominated by that of the motherboard.

For the current calibration there was a rather large pedestal (up to $10 \mathrm{GeV}$ ) due to clock coupling to the preamplifier inputs caused by the motherboard layout. On the hybrid itself, the clock feedthrough to the input is less than $100 \mathrm{MeV}$ (compared to $50 \mathrm{MeV}$ noise). After subtraction, the results (fig. 7) are similar to the voltage calibration, with an r.m.s. of $0.39 \%$, which is also dominated by the dispersion inside a given motherboard. There remains no correlation between high gain and high pedestal.

The ratio of the gains obtained with the two systems has an r.m.s. dispersion of $0.33 \%$, hinting at a common contribution due to the readout, presumably in the output leads and in the AXON cable. From this result it can be inferred that both systems are presumably within $0.2 \%$. 
The same kind of measurements has been performed directly on the calorimeter, on 192 channels on the front and middle compartment [12]. Raw data gave an r.m.s. of $0.7 \%$ in the front and $1 \%$ in the middle for both systems. As the 0T output amplitude is sensitive to the detector capacitance [13], a correction has been made for its $2 \%$ non uniformity. The r.m.s. value after correction is around $0.5 \%$ for both the front and the middle channels. The ratio of the two systems exhibits an r.m.s. dispersion of $0.5 \%$, again indicating a remaining contribution from a common factor.

The linearity of the two systems has been measured using the same setup [12]. The maximum input current corresponds to about $160 \mathrm{GeV}$ in one preamplifier, which is the highest energy in one cell attained in the beam tests. The results are very similar for the two systems with residuals smaller than $\pm 0.2 \%$. Measurements are under way to evaluate the linearity up to $1.5 \mathrm{TeV}$ ( $5 \mathrm{~mA}$ current in the calorimeter).

In summary the two systems have shown so far similar and good performance in terms of linearity as well as uniformity. The current calibration looks more attractive for a large system, although radiation tests have to be performed to assess that there is no leakage in the transistors, as it would be taken from the calibration current $I_{p}$. The voltage calibration is simpler and more radiation hard, but due to skin effects in the cables, the pulsers should be located close to the feedthrough so that the pulse shapes remain similar to the physics signals.

Tests will continue to be able to choose the most appropriate system.

\subsubsection{Timing and calibration procedure}

In each calorimeter channel the shaper response to the calibration pulse and to the physics signal produced by an incident particle must be sampled by the T\&H circuit as close as possible to its maximum. The narrow peak of the (fast) shaped response makes however the timing of the detector quite difficult, the desired accuracy being of the order of $1 \mathrm{~ns}$.

A two-fold procedure has been adopted. In the first step (timing of the calibration) the strobe to the T\&H modules was timed with respect to the peak of the calibration pulse. The peaking time of each channel was therefore measured by means of a computer-controlled delay box with an accuracy of a few tens of nanosecond. The speed of response of a channel depends on the performance of the front-end electronics and on the cell capacitance. The intrinsic peaking time dispersion of a given readout scheme is however rather modest ( $\sim 1 \mathrm{~ns})$ and the cell dimensions are quite uniform in each longitudinal sampling of a given readout block. Therefore average peaking times were first calculated inside the various blocks and samplings and the results were then equalized to the overall mean by inserting suitable delay cables. Finally the $\mathrm{T} \& \mathrm{H}$ strobe, common to the whole detector, was adjusted to sample the calibration signal at its (average) maximum.

In the second step (timing of the beam) the electronic chain was timed with respect to the incident particles by looking for the relative delay between the beam trigger and the $\mathrm{T} \& \mathrm{H}$ strobe which maximizes the calorimeter response to a physics signal, typically the reconstructed energy of an electromagnetic shower.

The timing of the detector, performed at the beginning of the data taking period, was then periodically monitored but no further adjustment was required.

Once the electronic chain was correctly timed, calibration coefficients were determined by fitting the ADC response of each channel to the test pulse with a third order polynomial in the energy range $0-160 \mathrm{GeV}$. The average sensitivity in electron energy scale is given in table 1 for the three readout schemes. The linearity is good, the second order correction amounting to $2 \%$ at $50 \mathrm{GeV}$. After calibration the residual channel-to-channel dispersion, i.e. the dispersion of the channel outputs when the same test pulse is injected at the inputs, was $0.2 \%$. The gain stability with time was found to be within $0.1 \%$ over a period of 60 hours.

The calibration procedure was repeated every few hours. 


\subsection{Electronic noise}

The electronic noise measured in the calorimeter, coming mainly from the preamplifier and therefore scaling as the cell capacitance, was in the range 40 to $70 \mathrm{MeV}$ per channel depending on the compartment and rapidity region. Similar values were found for the three front-end electronics (see table 1) when reported to the same cell capacitance (i.e. to the same $\eta$ ). In the first sampling the cell capacitances are low, the preamplifier noise is also low and the system noise contribution becomes no longer negligible. Therefore the given figures represent an upper limit.

A coherent noise at the level of $5 \%(10 \%)$ was also observed with the cold (warm) preamplifiers. This contribution is however small in the calorimeter area needed to reconstruct an electromagnetic shower (typically nine cells per compartment).

Table 1: Main parameters of the electronic chain

\begin{tabular}{|l|c|c|c|c|}
\hline & compartment & $\mathrm{Si}$ & GaAs & $0 \mathrm{~T}$ \\
\hline gain (MeV/count) & 1 & 40 & 40 & 45 \\
& 2 & 40 & 40 & 55 \\
& 3 & 45 & 45 & 60 \\
\hline incoherent noise (MeV) & 1 & 45 & 41 & 45 \\
at $\eta=0.2$ & 2 & 55 & 59 & 50 \\
& 3 & 70 & 72 & 65 \\
\hline coherent noise $(\mathrm{MeV})$ & 1,2 & 2.5 & 2.5 & 5.5 \\
\hline
\end{tabular}

\section{$3 \quad$ Test Beam Setup}

The 2 metre electromagnetic prototype was exposed to electron and pion beams at the H8 beam line facility of the CERN SPS in three different periods from autumn 1992 to autumn 1993. The hadronic calorimeter, which was located behind the electromagnetic module inside the same cryostat, allowed to study the response of a full LAr system to high energy hadrons. The relevant results are discussed in $[6,15]$.

The information from the hadronic module was also used to monitor the longitudinal containment of high energy electrons and to study the electron-hadron separation power of the two calorimeters.

The detectors were housed in a dedicated cryostat consisting of a $1.2 \mathrm{~mm}$ thick aluminium outer wall and of a $8 \mathrm{~mm}$ thick stainless steel inner vessel, separated by $30 \mathrm{~cm}$ of low density (Rohacell) foam. The total amount of material in front of the electromagnetic calorimeter, including the cryostat, a few centimetres of inactive liquid, cables and supports, was about $1 X_{0}$ at $\eta=0$.

The cryostat was mounted on a computer-controlled platform whose rotation in $\eta$ and $\phi$ allowed the beam to hit the prototype with the same incidence angles as particles originating from the LHC nominal interaction point, and to scan almost the full detector coverage. At the calorimeter front face the beam spot typically covered one cell $\left(\sim 2.5 \times 2.5 \mathrm{~cm}^{2}\right)$

The beam line was instrumented with four scintillation counters providing the trigger and with three multiwire proportional chambers used to extrapolate the particle trajectory to the calorimeter front face. The impact point could be estimated with an accuracy of about 300 $\mu \mathrm{m}$ in two orthogonal views. A threshold Čerenkov counter allowed to determine the electron contamination in the pion beam (see section 6 ).

The results discussed in this paper mainly refer to electron data in the energy range 10 to $287 \mathrm{GeV}$. Pions of 20,30 and $50 \mathrm{GeV}$ were also collected to study the $e / \pi$ separation capability of the calorimeter, while the signal-to-noise ratio for minimum ionizing particles was evaluated using the component of muons present in the electron beam at high energy. 
The electron showers were reconstructed in the first and second compartment of the calorimeter by summing the energy contained in an array of $3 \times 3$ cells centered around the channel with the highest signal. In the third sampling, which has a twice coarser $\eta$-granularity, only $2 \times 3$ cells in $\eta \times \phi$ were used. This cluster contains on average $\sim 90 \%$ of the shower energy, slightly decreasing with energy. A larger size array would allow for a better shower containment but would be more affected by the electronic noise and by the pile-up contribution at the LHC luminosity.

The electron position in the calorimeter was reconstructed in the two directions $(\eta, \phi)$ as the centre-of-gravity of the shower in the first sampling. When mentioning the particle position or impact point throughout this paper we will always refer to the shower barycentre reconstructed by the calorimeter in this way.

\subsection{Response dependence on the particle impact point}

This section explains the influence of the intrinsic Accordion geometry on the detector response, while the non-uniformity induced by the mechanics and by small calibration imperfections will be discussed in section 4.5 .

Figure 8 shows the variation of the calorimeter response with the electron position in $\eta$ over a large area. It can be seen that the energy deposition is maximum for particles hitting the centre of a cell and decreases towards the cell edges due to the reduced shower containment in the $3 \times 3$ cell cluster. This effect can be easily corrected for with a second order polynomial. At the border between two different kapton electrodes ( $\eta_{\text {cell }}=12.5$ in the figure) the response drop is more pronounced. This behaviour, which can be attributed in part to the wider nominal insulation region between the two border cells $(2 \mathrm{~mm}$ instead of $1 \mathrm{~mm})$ and in part to the shrinking of the readout boards in the cold, could be avoided with electrodes extending over a larger part of the calorimeter coverage. A polynomial correction was applied also in this case.

In the $\phi$ direction, perpendicular to the Accordion folds, small periodical variations in the traversed amount of LAr, inherent to the detector geometry [3], give rise to local changes in the sampling fraction. This results in a regular response modulation with peak-to-peak amplitude $\pm 1 \%$ and r.m.s. $0.5 \%$ (fig. 9). This modulation, which is essentially energy independent, was parametrized with a combination of a second order polynomial and a sinusoidal function. The residual r.m.s. after this correction is about $0.3 \%$.

The precision of the detector assembly was also studied by scanning the region at the boundary between two azimuthal modules of the stack. An example is shown in fig. 10. Away from the singular region the modulation described above is visible, but at the module edge a drop in the response of $2.5 \%$ is observed. This behaviour is consistent with the measurements of the double gap uniformity in the stack mentioned in section 2.3. As shown in fig. 4, at the module boundary the gap is $6-7 \%$ wider than elsewhere. Since in fast shaping conditions the collected charge depends on the gap thickness as $g^{-1 / 3}$ (for a constant operating voltage) the larger gap at the module edge is expected to result in a loss of charge of about $2 \%$, in good agreement with the data. Also in this case a correction could be applied.

\subsection{Response linearity}

The linearity and energy resolution were studied with sets of electron data taken in the energy range 10 to $287 \mathrm{GeV}$ in a few benchmark positions: $\eta=0.28$ and $\eta=0.90$ in the region read out with Si preamplifiers, and $\eta=0.50$ in the area equipped with GaAs hybrids.

The response variation with the impact point in $\eta$ and $\phi$ was corrected as described in the previous section by using the same set of parameters at all energies and in all positions. No correction for the longitudinal shower leakage in the hadronic calorimeter and for a weak energy dependence of the lateral shower containment was applied.

The response linearity was evaluated by comparing the mean values from Gaussian fits to the reconstructed energy spectra with the nominal beam energy for each momentum setting 
Table 2: Energy resolution fit parameters.

\begin{tabular}{|c|c|c|c|}
\hline & non pointing & \multicolumn{2}{|c|}{ 2 $\mathbf{m}$ prototype } \\
\cline { 2 - 4 } & $\eta=0$ & $\eta=0.28$ & $\eta=0.90$ \\
\hline$a\left(\% \mathrm{GeV}^{1 / 2}\right)$ & $9.84 \pm 0.24$ & $9.99 \pm 0.29$ & $10.42 \pm 0.33$ \\
$b(\mathrm{GeV})$ & $0.326 \pm 0.015$ & $0.282 \pm 0.017$ & $0.387 \pm 0.016$ \\
$c(\%)$ & $0.32 \pm 0.04$ & $0.35 \pm 0.04$ & $0.27 \pm 0.08$ \\
\hline
\end{tabular}

(fig. 11). The absolute energy scale was determined at $100 \mathrm{GeV}$. The two dashed lines in fig. 11 indicate the uncertainty (r.m.s.) on the nominal beam momentum

$$
\frac{\Delta(P)}{P}=\frac{25}{P} \% \oplus 0.5 \%
$$

where $P$ is in $\mathrm{GeV}$. The first term in this equation is related to hysteresis effects in the bending magnets, while the second term includes calibration and geometrical uncertainties. The apparently lower response of the calorimeter to $20 \mathrm{GeV}$ electrons, common to the three positions of incidence, is probably due to a beam momentum systematically lower than the nominal value. The error bars in the figure include an estimate of the beam rectifier stability $(4.5 \% / P)$ and the statistical error.

Taking into account that at $287 \mathrm{GeV}$ and small rapidity the average longitudinal leakage is about $0.5 \%$, it can be deduced that the calorimeter response is linear within $1 \%$ for all incidence points.

\subsection{Energy resolution}

The energy resolution measured with the 2 metre prototype is shown in fig. 12 . The results refer to electrons impinging at $\eta=0.28$ and $\eta=0.90$, both in the region equipped with $\mathrm{Si}$ preamplifiers. The data collected in the region read out with GaAs preamplifiers have not been used for this study due to a problem in the input stage of the hybrids (solved afterwards) which gave rise to a large electronic noise. After subtracting the contribution of the beam momentum spread $(0.3 \%$ at $287 \mathrm{GeV})$ the experimental points were fitted with the form

$$
\frac{\sigma(E)}{E}=\frac{a}{\sqrt{E}} \oplus \frac{b}{E} \oplus c
$$

where $a, b, c$ are the sampling, the noise and the local constant term respectively and $E$ is expressed in $\mathrm{GeV}$. The results of the fit are given in table 2.

The resolution at $\eta=0.28$ (almost orthogonal incidence) can be compared with the results obtained with a previous Accordion prototype with non pointing geometry [4], which are also given in table 2. Due to the identical sampling fraction and frequency of the two detectors, the measured stochastic terms are the same within the errors. The local constant term is also similar in the two cases, thus demonstrating that the Accordion concept can be adapted to a fully projective geometry without any loss in performance. This term can be attributed in part to the residual Accordion modulation on a local scale (over one cell) and in part to the fluctuations of the shower longitudinal containment which were estimated to be $0.25 \pm 0.05 \%$ at $287 \mathrm{GeV}$ and $\eta=0.28$. The electronic noise, about $300 \mathrm{MeV}$, is consistent with the value obtained from the ADC pedestal widths for an array of $3 \times 3$ towers.

The data collected at $\eta=0.90$, that is in the region with thinner lead in the absorber plates, were used to explore the calorimeter behaviour at large rapidity. The increase of the sampling term from $9.99 \%$ to $10.42 \%$ (table 2 ) is compatible with the prediction of the scaling law mentioned in section $2(10.23 \%)$, while with a constant absorber composition this term would become $11.7 \%$. The larger electronic noise is consistent with the increase in the cell capacitance $(C \propto 1 / \sin \theta)$. The better longitudinal containment of the shower at $\eta=0.9$, where the total 
thickness of the calorimeter is about $29 X_{0}$, might explain the slightly lower constant term. In conclusion the data demonstrate that the sampling term of the energy resolution can be kept under control as a function of rapidity by suitably reducing the density of the converter plates. This result however was obtained in a setup with a small amount of dead material in front of the calorimeter $\left(<1.5 X_{0}\right)$.

\subsection{Position resolution}

The space resolution of the calorimeter was determined by comparing the shower centreof-gravity, reconstructed in the calorimeter as explained in section 4, with the extrapolated electron impact point provided by the beam chamber system.

The systematic shift of the shower barycentre towards the cell centre, giving rise to an "S-shape" typical of pad detectors, is observed in the $\eta$ view but not in $\phi$, where the Accordion waves induce a better sharing of the shower energy among neighbouring cells [3]. The correct particle position in $\eta\left(\eta_{\text {true }}\right)$ was therefore obtained as

$$
\eta_{\text {true }}=a_{1}+a_{2} \arctan \left(\frac{\eta_{\text {calo }}-a_{3}}{a_{4}}\right)
$$

where $\eta_{\text {calo }}$ is the barycentre reconstructed in the calorimeter and the four parameters depend only weakly on the electron energy.

The position resolution measured in the two views at $\eta \simeq 0.28$ is shown in fig. 13 as a

function of the incident electron energy. By fitting the data with the expected $1 / \sqrt{E}$ law, where $E$ is expressed in $\mathrm{GeV}$, and after subtracting the contribution of the beam chambers, one gets

$$
\begin{aligned}
& \sigma_{\theta}=(0.210 \pm 0.015) \oplus \frac{4.70 \pm .05}{\sqrt{E}} \mathrm{~mm} \\
& \sigma_{\phi}=(0.186 \pm 0.021) \oplus \frac{3.87 \pm .05}{\sqrt{E}} \mathrm{~mm}
\end{aligned}
$$

which is in good agreement with the perfomance obtained with previous prototypes.

Finally figure 14 shows that in the $\eta$ view the resolution is better when the particle hits the calorimeter near the edge of a cell, thanks to a larger energy sharing with the neighbouring channels, than at the cell centre. The results quoted above were obtained over a spot covering about half a cell symmetrically around the cell centre.

\subsection{Uniformity of response}

The calorimeter behaviour over a relatively large area was determined by scanning 153 different cells with high energy electron beams. The aim of this study was to evaluate the response uniformity and the constant term of the energy resolution over a significant part of the prototype, including singular regions such as the boundaries between adjacent kapton boards or between azimuthal modules of the stack.

The response variation with the particle impact point was corrected as described in section 4.1 with a same set of parameters throughout the detector.

A few sources of response non-uniformity were identified in the offline analysis [16]. Three cells were most likely affected by a missing high voltage connection in one of their kapton electrodes and were removed from the data sample, even if the response could be in large part recovered with a suitable correction.

Some Si preamplifiers (about 10\% of the total) were found to be significantly slower (by $5-10 \mathrm{~ns}$ ) than the average. In these cases the channel calibration is even sensitive to timing differences between the calibration and the physics as small as 1 to $2 \mathrm{~ns}$. Since these slight differences are difficult to determine with accuracy, no correction could be applied.

Finally two of the $100 \Omega$ cables bringing the calibration pulse to the preamplifier motherboards exhibited a bad behaviour in the cold, resulting in a slower test pulse signal at the channel input and consequently in a wrong gain coefficient. The latter could be corrected by using measurements performed at the end of the beam test after warming up. 
All these problems have been solved for the next periods of data taking.

Figure 15 shows the resulting uniformity of response of the detector over the whole scanned region. It includes $287 \mathrm{GeV}$ electron data collected in the first two sectors of the calorimeter $(\eta<0.4$, Si and 0T electronics) and a sample of $197 \mathrm{GeV}$ electrons hitting the third sector $(0.4<\eta<0.6$, GaAs preamplifiers $)$. The resulting r.m.s. is $0.62 \%$. No relative normalization of the two energies was applied.

\subsection{Constant term of the energy resolution}

A large effort when designing and building the 2 metre prototype was devoted to minimizing the potential sources of non-uniformity deteriorating the detector energy resolution. According to the ATLAS specifications [1] a constant term of less than 1\% should be maintained over the full coverage of the electromagnetic calorimeter in order to explore physics channels of prime interest such as a possible $H \rightarrow \gamma \gamma$ decay.

Figure 16 shows the total energy spectrum for all the available $287 \mathrm{GeV}$ electron events. Data from 123 different cells in the $\mathrm{Si}$ and $0 \mathrm{~T}$ regions are included. The rather pronounced low energy tail can be attributed in part to longitudinal leakage outside the electromagnetic calorimeter and in part to the bad quality of the electron beam during the period in which the position scan was collected ${ }^{5)}$. This is confirmed by a comparison of the spectra obtained in a given cell in two data taking periods (fig. 17), with different beam conditions. It can also be seen that the material in the beam line affects significantly the left side of the distribution, whereas the effect on the spectrum width is negligible.

The fractional energy resolution obtained from a Gaussian fit to the spectrum in fig. 16 over the region not affected by the tail is $0.95 \%$ (beam momentum spread unfolded). By quadratically subtracting from this value the measured sampling and noise terms (table 2) as well as the estimated contributions from the shower longitudinal $(0.25 \%)$ and lateral $(0.15 \%)$ leakage one gets a constant term

$$
c=(0.69 \pm 0.05) \%
$$

This result is consistent with the quadratic sum of the local constant term $(\sim 0.27 \%$ from table 2 at $\eta=0.9$ where the longitudinal leakage is negligible) and the r.m.s. of the cell-to-cell response variation $(0.62 \%$, see section 4.5$)$, as expected. The same result was obtained from the data collected in the $\mathrm{GaAs}$ region with $197 \mathrm{GeV}$ electrons.

A major part of the observed constant term can be attributed to known instrumental effects. They are listed in table 3 . It can be seen that similar contributions come from inaccuracies in the calibration procedure and from imperfections in the detector mechanics.

In particular the last item in the table refers to the non-homogeneity in the thickness of the absorber plates shown in fig. 3. The effect on the calorimeter response can be inferred from fig. 18. The collected energy diminishes with increasing amount of lead in the hit cell, due to the decrease of the sampling fraction. It can be seen that the calibration accuracy reached is good enough to allow to observe the sensitivity of the detector even to relatively small changes $(0.5 \%)$ in the converter thickness.

The effects listed in table 3 , most of which realistically reducible, justify a large part of the constant term in expression (10). Other contributions, difficult to estimate, can come from abnormally slow preamplifiers or faulty calibration cables.

\section{$5 \quad$ Response to Muons}

Muons contaminating the high energy electron beam were used to study the prototype response to "minimum ionizing" particles. A clean muon sample was selected by applying cuts

\footnotetext{
5) The problem was originated by the presence of another experiment running in parasitic mode on the same
} beam line. 
Table 3: Expected contributions to the constant term

\begin{tabular}{|l|c|}
\hline EFFECT & contribution $(\%)$ \\
\hline Calibration & \\
\hline non-uniformity of calibration pulse & 0.2 \\
ADC $\rightarrow$ GeV conversion & 0.2 \\
timing precision & 0.2 \\
residual crosstalk & 0.14 \\
\hline Mechanics & \\
\hline residual $\phi$-modulation & 0.3 \\
gap non-uniformity & 0.15 \\
absorber thickness variation & 0.3 \\
\hline total $(\oplus)$ & 0.58 \\
\hline
\end{tabular}

on the energy deposited in the electromagnetic and the hadronic calorimeter so as to reject electron and pion events.

Since the Accordion geometry induces a sharing of the energy deposition between two contiguous cells in $\phi$ also in case of a minimum ionizing particle, the muon signal in the calorimeter was reconstructed by summing the energy from the two $\phi$ towers closest to the particle impact point. The latter was determined by using the information from the beam chambers to avoid any bias (the electronic noise is not negligible as compared to the muon signal in one channel).

The spectrum obtained in this way in the region read out with GaAs preamplifiers is plotted in fig. 19 together with the noise distribution, that is the energy contained in an area of the same size as the muon cluster $(2 \times 1$ cells in $\phi \times \eta$ and three samplings in depth) but far away from the beam position. The same energy scale as for electron data has been used. The most probable energy loss was determined by fitting the muon spectrum with a Moyal function [17] convoluted with a Gaussian. The resulting signal to noise ratio, defined as the ratio between the peak of the muon signal and the r.m.s. of the noise distribution, is four.

\section{$6 \quad e / \pi$ Separation}

The capability of the LAr electromagnetic and hadronic calorimeters to separate pions from electrons was studied with 20,30 and $50 \mathrm{GeV}$ beams [18]. This kind of study requires a very good knowledge of the beam contamination, which was obtained by using the information from the Cerenkov counter, by looking at the amount of energy deposited in the electromagnetic calorimeter and, in the case of pion beams, by putting a lead sheet $1 X_{0}$ thick in front of one of the scintillators equipping the beam line. The electron contamination in the pion beam was found to be $0.02 \%$ at $20 \mathrm{GeV}$ and $0.05 \%$ at $30 \mathrm{GeV}$, increasing to $(0.5 \pm 0.1) \%$ at $50 \mathrm{GeV}$. The $\mu$ content in the pion beam was estimated to be less than $2 \%$, while the pion contamination to the electron beam was $0.1 \%$ at $20 \mathrm{GeV}$.

The variables used to distinguish between electrons and pions are based on the longitudinal and lateral shower shape in the electromagnetic calorimeter and on the energy leakage in the hadronic section. Examples are shown in fig. 20. The pion rejection achieved, defined as the ratio between the initial number of pions and the number of pions surviving the identification cuts, is plotted in fig. 21 as a function of energy. At $50 \mathrm{GeV}$ the result represents a lower limit due to the non negligible electron contamination in the pion beam.

In the future ATLAS experiment [2] a better electron/hadron separation will be possible by combining the information from the calorimeter and from an inner tracker system and by using the $E / p$ ratio, where $E$ is the energy measured in the calorimeter and $p$ the momentum reconstructed in the tracker. 
The geometry, the readout and the calibration system of a large scale prototype of an Accordion electromagnetic calorimeter for LHC have been described in this paper. The detector performance, based on the analysis of electron, muon and pion data from several beam tests, can be summarized as follows:

- the sampling term of the energy resolution is $10 \% / \sqrt{E(\mathrm{GeV})}$ over a large rapidity coverage $(0<\eta<1)$.

- the detector uniformity of response measured over an area of $1 \mathrm{~m}^{2}$ is about $0.6 \%$.

- the overall constant term of the energy resolution amounts to $0.69 \pm 0.05 \%$ and can be in large part explained by known and reducible instrumental effects.

- with a cell size of $2.7 \mathrm{~cm}$ the space resolution is better than $4 \mathrm{~mm} / \sqrt{E(\mathrm{GeV})}$ in $\phi$ (perpendicular to the Accordion waves) and about $4.7 \mathrm{~mm} / \sqrt{E(\mathrm{GeV})}$ in $\eta$.

- the signal to noise ratio, measured with muons, is 4 .

Further tests with improved readout (new kapton electrodes, new preamplifiers, etc.) and calibration system are foreseen in the near future.

\section{Acknowledgements}

We are grateful to P.Bailly, L.Bonnefoy, L.Bonnemaison, C.Bourgeois, P.Brunel, P.Charra, Y.Cholley, M.Cighetti, J.Collon, P.Cornebise, R.Currat, S.Cuzon, G.Dubois-Dauphin, H.Elattar, A.Garagiola, N.Greguric, G.Guilhem, G.Gurrieri, P.Imbert, A.Jouenne, P.Lancon, B.Maulini, S.Mygelgard, B.Monticelli, D.Petiberghien, L.Pierron, P.Repain, F.Sabatini, G.Sannier, A. Sigrist and R.Voisin for their significant contribution.

\section{References}

[1] ATLAS Collaboration, Letter of Intent, CERN/LHCC/92-4.

[2] ATLAS Collaboration, Technical Proposal, CERN/LHCC/94-43.

[3] B. Aubert et al. (RD3 Collaboration), Performance of a liquid argon electromagnetic calorimeter with an Accordion geometry, Nucl. Instr. and Meth. A309(1991)438.

[4] B. Aubert et al. (RD3 Collaboration), Performance of a liquid argon Accordion calorimeter with fast readout, Nucl. Instr. and Meth. A321(1992)467.

[5] B. Aubert et al. (RD3 Collaboration), Performance of a liquid argon electromagnetic calorimeter with a cylindrical Accordion geometry, Nucl. Instr. and Meth. A325(1993)118.

[6] D.M.Gingrich et al. (RD3 Collaboration), Performance of a Liquid Argon Accordion Hadronic Calorimeter Prototype, CERN-PPE/94-127, submitted to Nucl. Instr. and Meth.

[7] M.Lefebvre, G.Parrour and P.Pétroff, Electromagnetic Liquid Argon Accordion Calorimeter Simulation, RD3 Internal Note 41 (unpublished).

[8] B. Yu and V. Radeka, BNL 52444(1990).

[9] D. Camin et al., Front-end in Gallium Arsenide, Nucl. Instr. and Meth. A315(1992)385.

[10] R.L. Chase et al., Transmission line connections between detector and front-end electronics in liquid argon calorimetry, Nucl. Instr. and Meth. A330(1993)228.

[11] C. de La Taille, Electronic noise in LAr calorimetry, RD3 Internal Note 45 (unpublished).

[12] C. de La Taille, Calibration in LAr calorimetry, RD3 Note in preparation.

[13] R.L. Chase et al., Experimental results on cable-coupled preamplifiers (0T), Nucl. Instr. and Meth. A343(1994)598.

[14] R.L. Chase et al., Calibration of liquid argon calorimeters at high luminosity, in Proceedings of the II Int. Conf. on Calorimetry, Capri 14-18 oct. 1991.

[15] M.Stipcevic, Thèse de Doctorat à l'Université de Savoie (19 Mai 1994), A study of a Hadronic Liquid Argon Calorimeter Prototype for an LHC experiment, LAPP-T-94/02 and RD3 Internal Note 62 (unpublished). 
[16] A. Cravero and F. Gianotti, Uniformity of response and energy resolution of a large scale prototype of the barrel Accordion calorimeter, ATLAS Internal Note CAL-NO-33 (unpublished).

S.Robertson and M.Lefebvre, Further test beam results on the electron energy resolution of the barrel Accordion calorimeter prototype, ATLAS Internal Note CAL-NO-49 (unpublished).

[17] J. E. Moyal, Phil. Mag. 46(1953)263.

[18] L.Fayard et al., Study of $e / \pi$ rejection with the RD3 LAr Accordion calorimter, RD3 Internal Note 50 (unpublished). 
Figure 1: View of the 2 metre Accordion prototype during assembly when only two azimuthal modules were mounted in the stack. 
Figure 2: Sketch of an elementary cell of the Accordion calorimeter. 
Figure 3: Thickness of the lead in the absorber plates as a function of the plate number in the stack. The vertical lines indicate the boundaries between azimuthal modules. 
Figure 4: Double gap capacitance $\left(C_{\text {gap }}\right)$, normalized to the overall average, as a function of the gap position in the stack for four different $\eta$ rows. 
Figure 5: Principle of operation of the voltage calibration. 
Figure 6: Principle of operation of the current calibration. 
Figure 7: Normalized amplitude of the calibration signals obtained on five motherboards with the voltage (top) and the current (bottom) system. 
Figure 8: Normalized calorimeter response as a function of the electron impact point in $\eta$ for a $287 \mathrm{GeV}$ incident beam. The arrows indicate the centre and the edge of a cell. The full line is a fit to guide the eye. 
Figure 9: Normalized calorimeter response as a function of the particle impact point in $\phi$ for electrons of various energies. 
Figure 10: Normalized response as a function of $\phi$ for $287 \mathrm{GeV}$ electrons hitting the calorimeter near the boundary between the two upper modules of the stack. The dashed curve is a fit to the energy response away from the singular region. 
Figure 11: Calorimeter response normalized to the incident beam energy (the ratio is fixed to 1 at $100 \mathrm{GeV}$ ) as a function of the electron beam energy in three positions of incidence. The dashed lines are explained in the text. 
Figure 12: Fractional energy resolution as a function of the incident beam energy for electrons hitting the calorimeter at $\eta=0.28$ (closed circles) and $\eta=0.90$ (open circles). The beam momentum spread has been unfolded. The full and dashed lines are the best fits to the experimental points (see text). 
Figure 13: Position resolution of the calorimeter in $\eta$ (open circles) and $\phi$ (closed circles) as a function of the electron beam energy. The contribution of the beam chambers $(\sim 300 \mu \mathrm{m})$ has not been unfolded. The lines are fit to the experimental points (see text). 
Figure 14: Position resolution of the calorimeter in the $\eta$ direction for $30 \mathrm{GeV}$ electrons as a function of the particle distance from the cell centre. The curve is a fit to the experimental points. 
Figure 15: Average response, normalized to the incident beam energy, reconstructed in the calorimeter for high energy electrons as a function of the hit cell in $\eta$ for different $\phi$ rows. The vertical lines indicate the boundaries between adjacent kapton boards. 
Figure 16: Overall energy spectrum reconstructed in the calorimeter for $287 \mathrm{GeV}$ electrons hitting 123 cells of the prototype (about 150000 events in total). The full line is a Gaussian fit. 
Figure 17: Energy spectra reconstructed in the calorimeter for $287 \mathrm{GeV}$ electrons hitting the same cell. Data were taken with bad (open circles) or good (closed circles) beam conditions. The two spectra are normalized to the same number of events. 
Figure 18: Calorimeter response, normalized to the average, to high energy electrons as a function of the total lead thickness (normalized to the overall average) in the hit cell. Each point is integrated in $\eta$. 
Figure 19: Total signal produced in the calorimeter by $300 \mathrm{GeV}$ incident muons (closed circles) in electron energy scale. The grey histogram is the Monte Carlo absolute prediction while the full line is the distribution of the electronic noise. 
Figure 20: Fraction of energy released in the third compartment of the Accordion (top) and width of the shower (bottom) for electrons (full line) and pions (dashed line) of $20 \mathrm{GeV}$. The events at zero consist of pions non interacting in the electromagnetic calorimeter. 
Figure 21: Pion rejection for 95\% electron efficiency obtained with the electromagnetic and hadronic calorimeter as a function of the incident beam energy. 